\title{
Feminizing Wolbachia in an insect, Ostrinia furnacalis (Lepidoptera: Crambidae)
}

\author{
D Kageyama, G Nishimura, S Hoshizaki and Y Ishikawa \\ Laboratory of Applied Entomology, Department of Agricultural and Environmental Biology, Graduate School of Agricultural and \\ Life Sciences, University of Tokyo, Japan
}

\begin{abstract}
Wolbachia, which forms a group of maternally inherited bacteria in arthropods, often cause reproduction alterations in their hosts, such as cytoplasmic incompatibility, parthenogenesis, male-killing, hybrid breakdown and feminization. To date, Wolbachia-induced feminization has been reported only in isopods. Here we report that a Wolbachia strain feminizes an insect host, Ostrinia furnacalis. Among 79 wild females of $O$. furnacalis examined, Wolbachia infection was detected in 13 females. Twelve of the 13 infected females produced all-female progenies, and this trait was maternally inherited. Tetracycline treatment of thelygenic matrilines resulted in the production of all-male progenies. The present
\end{abstract}

findings indicate that the Wolbachia infection induces feminization of genetic males in $O$. furnacalis. Differences in the Wolbachia-induced feminization in $O$. furnacalis and that in isopods are discussed along with the differences in sex determination mechanisms between insects and isopods. Phylogenetic analysis of the wsp gene sequence of Wolbachia suggests independent evolutionary origins for the Wolbachia-induced feminizations in $O$. furnacalis and in isopods. Our findings over 5 years suggest that the infection has been maintained at a low prevalence in the $O$. furnacalis population.

Heredity (2002) 88, 444-449. DOI: 10.1038/sj/hdy/6800077

Keywords: feminization; Lepidoptera; Ostrinia furnacalis; sex-ratio distorter; Wolbachia

\section{Introduction}

Much attention has been increasingly paid to selfish genetic elements such as Wolbachia (eg, Werren et al, 1988; O'Neill et al, 1997). Wolbachia are a group of cytoplasmically transmitted bacteria prevailing widely among arthropods (eg Stouthamer et al, 1999). Wolbachia are primarily of interest because they can cause alterations to the reproduction of their hosts; male-killing (eg, Hurst et al, 1999), thelytokous parthenogenesis (eg, Stouthamer et al, 1993), feminization of genetic males (eg, Rousset et al, 1992), hybrid breakdown (Vala et al, 2000) and cytoplasmic incompatibility (eg, O'Neill et al, 1992). Wolbachia may have promoted rapid speciation in arthropods (Bordenstein et al, 2001).

The Asian corn borer, Ostrinia furnacalis (Lepidoptera: Crambidae) is a major pest of Zea mays in eastern and southeastern Asia. Miyahara (1984) first reported the occurrence of female-biased sex ratio (thelygeny) in Japanese populations of O. furnacalis, but its mechanism and causal agent were not identified. Kageyama et al (1998) found that thelygeny was inherited in a matriline of $O$. furnacalis and that feminization of genetic males was caused by a bacterial infection. However, the causal bacteria of the feminization in $O$. furnacalis remained unidentified.

Correspondence: S Hoshizaki, Laboratory of Applied Entomology, Department of Agricultural and Environmental Biology, Graduate School of Agricultural and Life Sciences, University of Tokyo, Japan.

E-mail:ahossy@mail.ecc.u-tokyo.ac.jp

Received 24 July 2001; accepted 18 February 2002
In the present study, we reveal that the feminization of genetic males in O. furnacalis is caused by infection with a Wolbachia strain, and estimate the phylogenetic position of the Wolbachia strain based on the wsp gene sequence. This is the first study to report the occurrence of a feminizing Wolbachia in insects.

\section{Materials and methods}

Insects

We collected female adults of O. furnacalis from Matsudo (Chiba pref., Japan) in the summers of 1996-2000. Some of the findings of the present study, for insects collected in 1996, has previously been published by Kageyama et al (1998).

The captured females were individually allowed to oviposit in the laboratory. Most of the collected females laid fertile eggs within a few days. Larvae were reared by broods on an artificial diet (Silkmate 2M, Nihon-Nosan, Yokohama, Japan). At the pupal stage, insects were sexed based on the abdominal tip morphology. A piece of cotton soaked with $3 \%$ sucrose was provided for adult moths. Insects were reared under the conditions of $23^{\circ} \mathrm{C}$ and 15L/9D. Twenty females and 20 males were put in a mating cage, and 2 days later, females were separately allowed to oviposit. After oviposition, ovaries of females were dissected and stored in STE buffer ( $\mathrm{O}^{\prime} \mathrm{Neill}$ et al, 1992) at $-20^{\circ} \mathrm{C}$ until extraction of DNA.

Maternal inheritance of female-biased sex ratio When a wild female produced a thelygenic family $(P<$ 0.01 , chi-squared test), daughters were used to found a 
matriline. Matrilines were maintained by crossing females with males from normal lines. A normal line was a pool of matrilines with the parental sex ratio not significantly distorted from 1:1.

\section{Tetracycline treatment}

Tetracycline hydrochloride was mixed into the larval diet at $0.06 \%$ wet weight and fed to the larvae from the neonate stage.

The tetracycline treatment was used to check whether the thelygeny was due to bacteria-induced feminization. Lepidopteran insects have been suggested to have either ZW/ZZ or ZO/ZZ sex chromosomes (Traut and Marec, 1996), and this was also assumed for O. furnacalis. If the production of all-male offspring occurs after antibiotic elimination of the feminizer, it indicates feminization of a genetic male, since the genotype of their mother should be ZZ to produce ZZ eggs exclusively. As another possibility, if a male-killing bacterium is causing the thelygeny, a 1:1 sex ratio should result from the tetracycline treatment.

\section{PCR assay of Wolbachia infection}

One of the pair of ovaries in a female adult was ground in $100 \mu \mathrm{l}$ of STE buffer (O'Neill et al, 1992) with $2 \mu \mathrm{l}$ of proteinase $\mathrm{K}(20 \mathrm{mg} / \mathrm{ml}), 1 \mu \mathrm{l}$ of 2 -mercaptoethanol and $10 \mu \mathrm{l}$ of $10 \%$ SDS. The homogenate was incubated at $37^{\circ} \mathrm{C}$ for at least $30 \mathrm{~min}$ and at $95^{\circ} \mathrm{C}$ for $5 \mathrm{~min}$. The lysate was extracted with phenol-chloroform and chloroform once each, and DNA was precipitated with ethanol. The DNA pellet was dissolved in $50 \mu \mathrm{l}$ of TE buffer.

Polymerase chain reactions (PCR) specific to the Wolbachia 165 rDNA gene were conducted in $10 \mu$ l reaction volumes including $1 \mu \mathrm{l}$ of DNA samples following O'Neill et al. (1992).

Sequencing and phylogenetic analysis

A PCR specific to the wsp gene of Wolbachia was performed (Zhou et al, 1998). The PCR products were purified using the GENE CLEAN III kit (Bio101, La Jolla, CA, USA), and both DNA strands were directly sequenced using the ABI 377 DNA sequencer with the BigDye terminator cycle sequencing kit (Perkin Elmer).

The wsp sequence of Wolbachia infecting O. furnacalis was initially aligned to 29 wsp sequences of other strains of B-group Wolbachia and two outgroup sequences from A-group Wolbachia (the Wolbachia strains from Drosophila melanogaster and D. sechellia) using the program package CLUSTAL W ver1.5 (Thompson et al, 1994), and the alignment was manually modified based on the inferred amino acid sequences using the sequence alignment editor BioEdit (Hall, 1999). The third hypervariable region (positions 518-565) was excluded from the analyses (Zhou et al, 1998). The molecular phylogenetic tree was estimated by the maximum likelihood method using the program package PAUP* (Swofford, 1996). We employed the general time reversible (GTR) model with discretegamma approximation (four rate categories). All model parameters were estimated from the data using a starting tree topology that had previously been inferred with unweighted maximum parsimony. These parameter estimates and the starting tree topology were then employed for a heuristic search based on tree bisection and reconnection (TBRs). Bootstrap analysis was done with 100 replications.

\section{Results}

Wolbachia infection and female-biased sex ratio

Three of 13 females collected in the field in 1996 were thelygenic (Figure 1). We found Wolbachia infection in two thelygenic females (M9 and M11, Table 1), but we did not examine infection in the other 11 wild females (Kageyama et al, 1998).

Wolbachia infection was found in 11 of 79 females collected in the field during 1997-2000 (Figure 1). Throughout the 4 years, the frequencies of infected females were rather low $(0-43 \%)$. Ten of the 11 infected females produced all-female offspring (strong thelygeny), while no uninfected females produced the strongly thelygenic offspring (Table 1, Figure 1). One infected female (MD771)
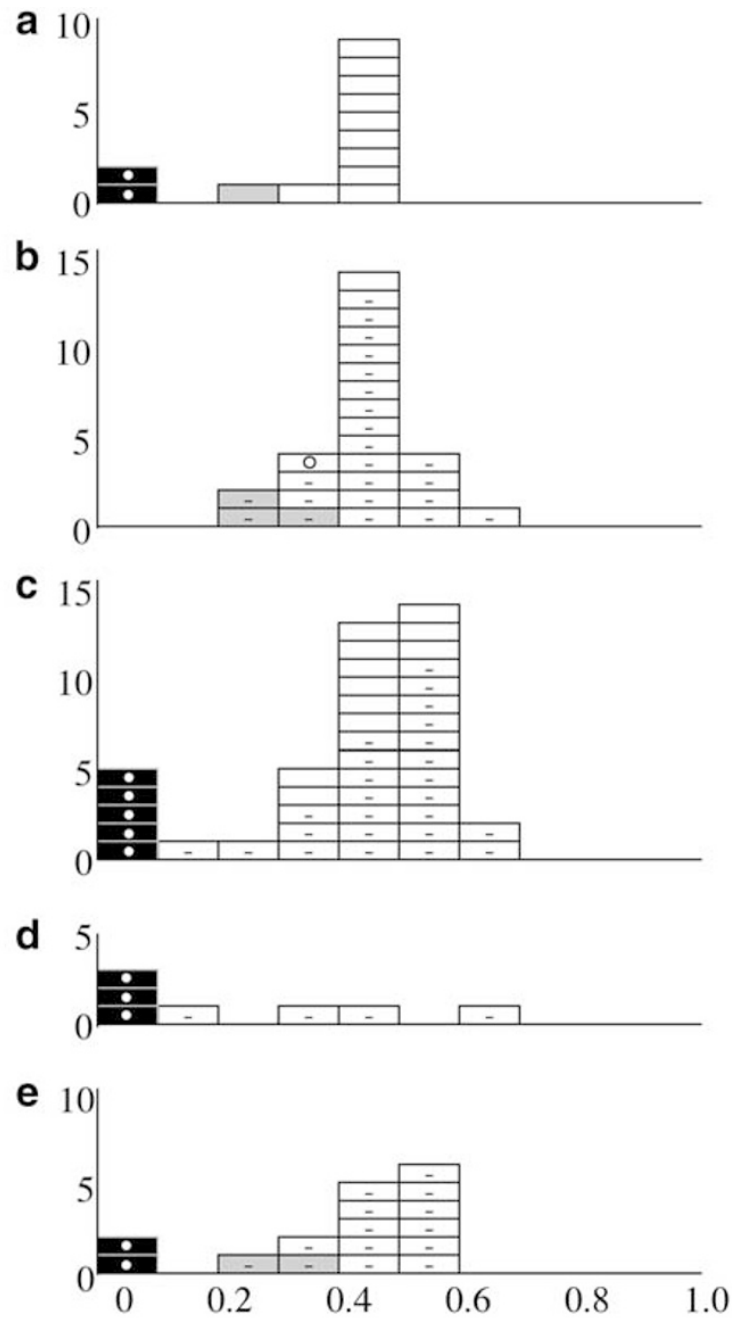

\section{Proportion male}

Figure 1 Distribution of sex ratio in progenies produced by wild females of Ostrinia furnacalis collected at Matsudo in (a) 1996, (b) 1997, (c) 1998, (d) 1999 and (e) 2000. Boxes with circles and minus signs indicate Wolbachia-infected and uninfected broods, respectively. Broods without these signs were not examined for Wolbachia infection. Black and grey boxes indicate strong and weak SR broods respectively. Two broods with size less than 10 in (c) and (d) each were excluded from the figure. 
Table 1 Wolbachia infection and sex ratios of thelygenic matrilines (strongly and weakly) of Ostrinia furnacalis with egg hatch rates in parental families

\begin{tabular}{|c|c|c|c|c|c|c|c|c|c|c|}
\hline \multirow[t]{2}{*}{ Line } & \multirow{2}{*}{$\begin{array}{l}\text { Wolbachia } \\
\text { infection }\end{array}$} & \multicolumn{2}{|c|}{ Parental } & \multirow{2}{*}{$\begin{array}{l}\text { Egg hatch } \\
\text { rate }\end{array}$} & \multicolumn{2}{|c|}{$F_{1}$} & \multicolumn{2}{|c|}{$F_{2}$} & \multicolumn{2}{|c|}{$F_{3}$} \\
\hline & & Female & Male & & Female & Male & Female & Male & Female & Male \\
\hline \multicolumn{11}{|c|}{ (a) Strongly thelygenic matrilines } \\
\hline \multirow[t]{2}{*}{ M9* } & + & 89 & 0 & ne & 184 & 27 & $\begin{array}{r}15 \\
9\end{array}$ & $\begin{array}{l}0 \\
0\end{array}$ & & \\
\hline & & & & & 22 & 7 & 81 & 0 & & \\
\hline M11* & + & 29 & 0 & ne & 56 & 0 & 37 & 8 & & \\
\hline \multirow{4}{*}{ MD804 } & + & 38 & 0 & 0.97 (123) & 43 & 0 & 65 & 0 & 43 & 0 \\
\hline & & & & & & & & & 55 & 0 \\
\hline & & & & & & & & & 36 & 0 \\
\hline & & & & & & & & & 65 & 0 \\
\hline \multirow[t]{2}{*}{ MD807 } & + & 32 & 0 & $0.96(97)$ & 35 & 0 & & & & \\
\hline & & & & & 34 & 0 & & & & \\
\hline MD825 & + & 31 & 0 & $0.81(392)$ & 43 & 0 & & & & \\
\hline \multirow[t]{2}{*}{ MD826 } & + & 25 & 0 & ne & 20 & 0 & & & & \\
\hline & & & & & 31 & 0 & & & & \\
\hline MD846 & + & 42 & 0 & 0.95 (186) & 45 & 0 & 45 & 0 & 10 & 1 \\
\hline MD903 & + & 48 & 0 & ne & 51 & 0 & & & & \\
\hline \multirow[t]{2}{*}{ MD910 } & + & 58 & 0 & ne & 82 & 3 & 3 & 10 & & \\
\hline & & & & & & & 2 & 2 & & \\
\hline \multirow[t]{2}{*}{ MD920 } & + & 28 & 0 & ne & 44 & 0 & & & & \\
\hline & & & & & 43 & 0 & & & & \\
\hline \multirow[t]{6}{*}{ MD030 } & + & 14 & 0 & ne & 48 & 0 & 43 & 0 & 26 & 0 \\
\hline & & & & & 51 & 0 & 29 & 0 & 25 & 0 \\
\hline & & & & & & & & & 32 & 0 \\
\hline & & & & & & & & & 21 & 0 \\
\hline & & & & & & & & & 18 & 0 \\
\hline & & & & & & & & & 33 & 0 \\
\hline \multirow[t]{4}{*}{ MD049 } & + & 47 & 0 & ne & 30 & 0 & 43 & 0 & 20 & 0 \\
\hline & & & & & 56 & 0 & 49 & 0 & 12 & 0 \\
\hline & & & & & & & & & 7 & 0 \\
\hline & & & & & & & & & 9 & 0 \\
\hline \multicolumn{11}{|c|}{ (b) Weakly thelygenic matrilines } \\
\hline $\mathrm{M}^{3} 3^{*}$ & - & 22 & 6 & ne & & & & & & \\
\hline MD743 & - & 94 & 48 & ne & & & & & & \\
\hline MD745 & - & 39 & 11 & ne & 13 & 8 & & & & \\
\hline MD747 & - & 44 & 16 & ne & & & & & & \\
\hline \multirow[t]{2}{*}{ MD003 } & - & 59 & 29 & $1.00(204)$ & 16 & 12 & 34 & 33 & & \\
\hline & & & & & & & 3 & 11 & & \\
\hline MD0037 & - & 31 & 10 & ne & & & & & & \\
\hline
\end{tabular}

Wild females were checked for Wolbachia infection by PCR. Egg hatch rates are given with total numbers of eggs in parentheses. A large number of eggs were used to check their hatchability, and not necessarily all of the hatched larvae were inoculated on the diet to avoid overcrowding. ne, not examined. *Sex ratio data on lines M9, M11 and M13 are from Kageyama et al, 1998.

produced a brood with a sex ratio not strongly distorted (22 females and 10 males, $P=0.03$ by chi-squared test). Six wild females that were not infected produced families with significantly female-biased sex ratios $(P<0.01$ by chi-squared test), but these families included males at more than $20 \%$ (defined as weak thelygeny, Table 1, Figure 1) in contrast to the strong thelygeny seen in the Wolbachia-infected lines. In addition, two wild uninfected females produced families that appeared to be biased to female (10 females and two males, $P<0.05$; 13 females and three males, $P<0.05$; Figure 1).

The strong thelygeny was maternally inherited for all of the seven matrilines examined albeit with four exceptional cases (Table 1). Two $\mathrm{F}_{2}$ broods (three females and 10 males, and two females and two males) in matriline MD910 were not biased towards females. One $F_{1}$ brood (22 females and seven males) in matriline M9 and the $F_{2}$ brood (37 females and eight males) in matriline M11 showed rather weak thelygeny, although they are sig- nificantly female-biased ( $P<0.01$ by chi-squared test). On the other hand, the weak thelygeny disappeared in subsequent generations for both of the two matrilines examined (Table 1). We could not obtain offspring from the MD771 female.

Thus, Wolbachia infection was strongly associated with the all-female production in O. furnacalis females.

The mechanism of strong thelygeny

The average egg hatch rate for four families of strong thelygeny was 0.923 (Table 1), indicating that the Wolbachia-associated thelygeny was not due to early malekilling. All the larvae fed with tetracycline developed into females in all of seven matrilines of strong thelygeny, but these female adults produced all-male progenies (Table 2). The PCR assay confirmed that Wolbachia was eliminated from ovaries of tetracycline-treated females. These findings strongly suggest that the Wolbachia infection caused the strong thelygeny through feminization of 
Table 2 Sex ratios of broods produced from tetracycline-treated strongly thelygenic females

\begin{tabular}{lccc}
\hline Line & Replicate & Female & Male \\
\hline M11* & a & 0 & 24 \\
& b & 0 & 59 \\
& c & 0 & 58 \\
& d & 0 & 24 \\
& e & 0 & 50 \\
MD804 & f & 0 & 8 \\
MD826 & & 0 & 91 \\
MD846 & & 0 & 70 \\
MD920 & & 0 & 46 \\
MD030 & & 0 & 51 \\
MD049 & & 0 & 29 \\
& & 0 & 51 \\
& a & 0 & 49 \\
\hline
\end{tabular}

*Data on line M11 are from Kageyama et al, 1998.

genetic males in O. furnacalis. Namely, Wolbachia feminizes individuals carrying $\mathrm{ZZ}$ sex chromosomes (male genome), and such feminized individuals solely produce $\mathrm{ZZ}$ eggs that develop into male adults in the absence of the Wolbachia.

If Wolbachia-infected genetic females ( $\mathrm{ZW}^{*}$, an asterisk indicates Wolbachia infection) were included among the strongly thelygenic females in addition to the feminized genetic males $\left(\mathrm{ZZ}^{*}\right)$, they would have produced broods with normal sex ratio after the tetracycline treatment. In the present study, however, no such female was found (Table 2). The present finding can be explained if we assume that the infection of Wolbachia is old; Since $\mathrm{ZZ}^{*}$ females produce solely $Z Z^{*}$ females while $Z W^{*}$ females produce $Z^{*}$ and $Z^{*}$ females, the proportion of $Z W^{*}$ females will decrease sharply, and eventually almost all of the Wolbachia-bearing females will be $\mathrm{ZZ}^{*}$, provided that the fitness of $\mathrm{ZZ}^{*}$ and $\mathrm{ZW}^{*}$ females is similar.

\section{Molecular phylogenetic affiliation of the Wolbachia strain in $O$. furnacalis}

Nucleotide sequences of a portion (555 bp) of the wsp gene of Wolbachia in the 13 infected O. furnacalis females were determined. All the 13 sequences were identical (DDBJ/EMBL/GenBank nucleotide sequence databases with the accession number AB056664), suggesting infection with an identical strain of Wolbachia.

Molecular phylogenetic analyses have revealed the occurrence of two major clades (A and B) in Wolbachia strains infected with arthropods (Werren et al, 1995; van Meer et al, 1999; see also Vandekerckhove et al, 1999). The present analysis of wsp sequences showed that the Wolbachia strain in $O$. furnacalis is a member of the B group (Figure 2). Within the B group, the Wolbachia in O. furnacalis did not have a sister relationship to the feminizing Wolbachia strains in isopods, Armadillidium vulgare, Oniscus asellus and Porcellio scaber.

\section{Discussion}

In lepidopteran insects, thelygenies due to early (embryonic) male-killing have been known, some of which are caused by Wolbachia infection (Hurst et al, 1999; Jiggins et al, 2000, and references therein). In O. furnacalis, however, two lines of evidence indicated that Wolbachia

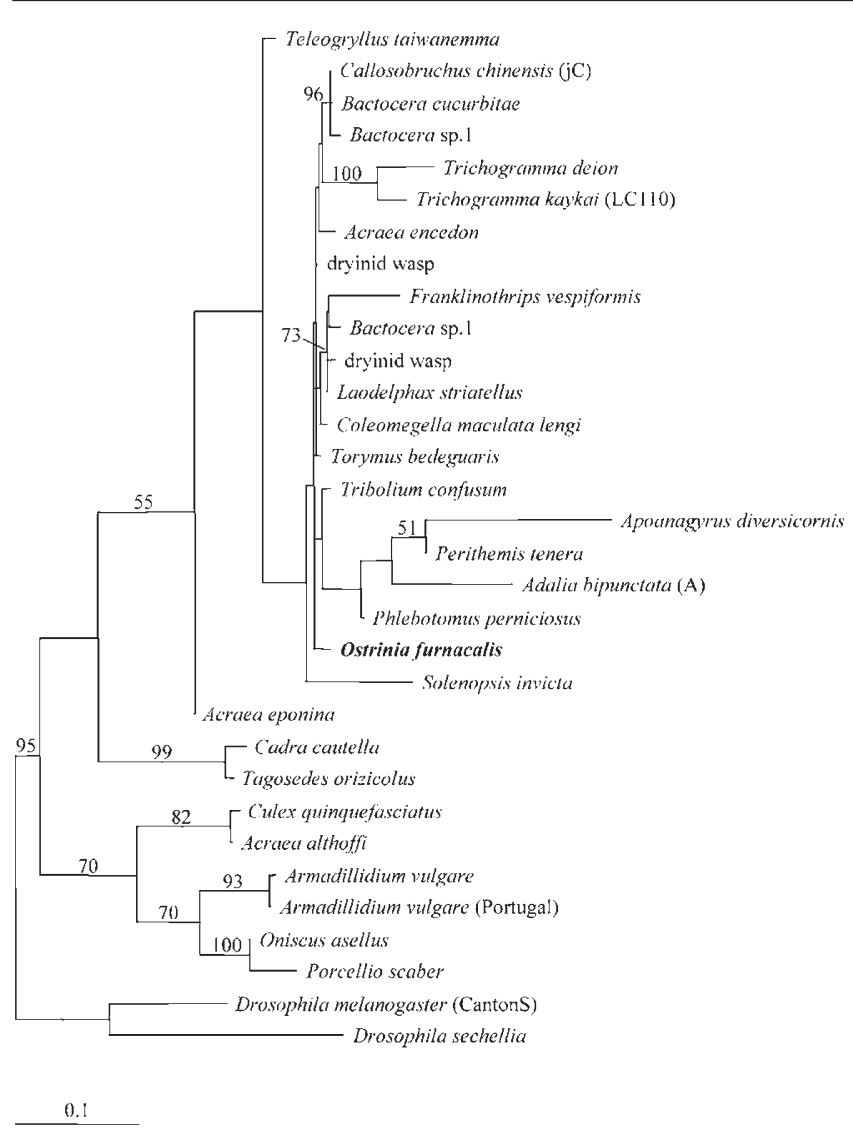

Figure 2 Phylogenetic tree of B-group Wolbachia based on wsp gene sequence data with two outgroups of A-group Wolbachia from Drosophila sechellia and D. melanogaster. Wolbachia strains are given as their host species names. The tree was constructed by the maximum likelihood methods using PAUP* (Swofford, 1996). The tree has log likelihood of -4136.75 . The results of 100 bootstrap replicates are shown above the branches. GenBank accession numbers of included published sequences are as follows: AB035514, AB038326, AB039284, AB045314, AB046720, AF020060, AF020065, AF020073, AF020076, AF020080, AF020083, AF020084, AF020085, AF071915, AF071916, AF071917, AF071927, AF217720, AF217725, AF237884, AF243436, AF295346, AF295347, AF295348, AJ130714, AJ130716, AJ269474, AJ269475, AJ269476, AJ271194, AJ271197.

infection causes feminization of genetic males. First, Wolbachia infection was strongly correlated to the strong thelygeny: this trait was found in 12 of 13 wild Wolbachiainfected females, but not in 68 uninfected females. Second, the result of antibiotic elimination of Wolbachia infection strongly suggested that feminization of genetic males underlay the strong thelygeny, although an alternative interpretation of the result is possible, as discussed below. This is the first study to report the occurrence of Wolbachia-induced feminization in insects, which has been known only from several isopod species (Rigaud et al, 1997).

It is possible to interpret the all-male production after antibiotic elimination of Wolbachia in a more complicated way; First, Wolbachia-infected females of O. furnacalis are assumed to be not viable without infection. This 'obligatory infection' hypothesis, in combination with a further hypothesis that Wolbachia causes male-killing during the larval stage (late male-killing), can explain the strong thelygeny in $O$. furnacalis. However, although a case of obligatory infection was recently reported in a parasitic 
wasp (Dedeine et al, 2001), Wolbachia have never been demonstrated to be obligatory in any other host species. Moreover, late male-killing due to Wolbachia infection has not been found in any animal to date. Taken together, it is very unlikely that the Wolbachia-induced strong thelygeny in $O$. furnacalis is caused by 'obligatory infection and late male-killing'.

Among the 13 Wolbachia-infected females of O. furnacalis, one female (MD771) produced non-thelygenic progeny (22 females and 10 males, $P>0.01$ ). Three explanations are possible for this exceptional case. First, the MD771 female may have harboured a Wolbachia strain different from the other 12 infections, although it was not distinguishable in terms of the wsp sequence. Secondly, the MD771 female may have failed to transmit the infection to some of the eggs. Lastly, the density of Wolbachia in MD771 females may have been low, leading to failure in feminizing some of the progeny. In addition, the latter two explanations might be relevant to the two nonthelygenic broods in matriline MD910 and the two weakly thelygenic broods in matrilines M9 and M11 (Table 1).

Wolbachia-induced feminization is known in isopods such as Armadillidium vulgare (Rigaud et al, 1997 for a review). In comparison, two phenotypic differences were found between the feminizations in A. vulgare and $O$. furnacalis. First, Wolbachia-infected lines of $A$. vulgare occasionally produce intersexes, which has not been found in O. furnacalis. Second, young females of Wolbachia-infected $A$. vulgare, when reared at $30^{\circ} \mathrm{C}$ for eliminating the infection, progressively acquired the male phenotype within the treated generation. In O. furnacalis, however, tetracycline did not change the sex of individuals in the treated generation, and all-male offspring was produced in the subsequent generation.

These distinct phenotypic characteristics of feminization are most likely to be relevant to differences in sex determination and/or differentiation processes between A. vulgare and O. furnacalis. In A. vulgare, a 'male gene' has been suggested to control development of the androgenic gland that produces androgenic hormone. The androgenic hormone triggers the male differentiation after the fourth moult. Wolbachia may affect the activity of the male gene (Rigaud et al, 1997).

In insects, the sex determination process has been well elucidated only in D. melanogaster (for a recent review, Schütt and Nöthiger, 2000). The sex in Drosophila is determined at the embryonic stage, and is not affected by diffusing substances such as sex hormones in the later developmental stages. This mechanism is also supported for insects other than Diptera (eg, Hoy, 1994; but see also De Loof and Huybrechts, 1998). In congruence with this widely accepted notion, tetracycline-treated larvae of infected $O$. furnacalis developed into female adults, suggesting that the feminizing action of Wolbachia operates at the embryonic stage. The target(s) of the feminizing action may be some molecule(s) that play a role in sex determination during the embryonic stage of $O$. furnacalis, although the molecular mechanism of sex determination in Lepidoptera has not been elucidated. The silkworm Bombyx mori possesses a homolog of $d s x$, which is one of the Drosophila sex determination genes (Ohbayashi et al, 2001). The $d s x$ homolog in B. mori, as well as $d s x$ in Drosophila, is subjected to sex-specific splicing.

On the maximum likelihood tree of Wolbachia strains based on wsp sequences, the feminizing Wolbachia in Ostrinia does not have a sister relationship with that in A. vulgare, while the Wolbachia strains in isopods were monophyletic. This suggests that the evolutionary origins of feminization were independent in $A$. vulgare and $O$. furnacalis, provided that there has been no recombination between Wolbachia strains (but see Werren and Bartos, 2001).

Feminization mediated by a cytoplasmic parasite such as Wolbachia can increase the frequency of the parasite in the host population (O'Neill et al, 1997). However, our observation of Wolbachia infection in the Matsudo population of $O$. furnacalis during 5 years (Figure 1) did not show that the frequency of infected moths were increasing, but rather strongly suggested that the infection was maintained at a low prevalence. In isopods, the presence of nuclear gene(s) that resist to the vertical transmission or feminizing activity of Wolbachia has been argued (Rigaud and Juchault, 1992; Juchault et al, 1994). A similar nuclear resistance gene may explain the low prevalence of Wolbachia infection in O. furnacalis. However, such a resistance factor in $O$. furnacalis, if present, appears not to be functioning, at least under the laboratory conditions, because the rates of vertical transmission of Wolbachia were high and Wolbachia infection induced strong, not weak, thelgeny in O. furnacalis (Table 1, Figure 1). As another possibility, sexual selection against the feminized genetic males may be preventing the spread of Wolbachia infection in the $O$. furnacalis population, as has been argued in the case of $A$. vulgare (Moreau et al, 2001); however, this hypothesis must be critically tested through future studies.

\section{Acknowledgements}

We thank Yongping Huang and Takuma Takanashi for insect rearing, Tetsuhiko Sasaki, Toru Shimada, Kazuki Miura and Masaru Tamura for stimulating discussions and Sadahiro Tatsuki for continuous encouragement. This work was supported by a grant-in-aid for Scientific Research from the Ministry of Education, Sciences, Sports and Culture of Japan (09839010) to SH and JSPS fellowship for young scientists to DK.

\section{References}

Bordenstein SR, O'Hara FP, Werren JH (2001). Wolbachiainduced incompatibility precedes other hybrid incompatibility in Nasonia. Nature 409: 707-710.

Dedeine F, Vavre F, Fleury F, Loppin B, Hochberg ME, Boulétreau M (2001). Removing symbiotic Wolbachia bacteria bacteria specificially inhibits oogenesis in a parasitic wasp. Proc Natl Acad Sci USA 98: 6247-6252.

De Loof A, Huybrechts R (1998). "Insects do not have sex hormones": a myth? Gen Compar Endocrinol 111: 245-260.

Hall TA (1999). BioEdit: a user-friendly biological sequence alignment editor and analysis program for Windows 95/98/NT. Nucl Acids Symp Ser 41: 95-98.

Hoy MA (1994). Insect Molecular Genetics, Academic Press.

Hurst GDD, Jiggins FM, Von der Schulenburg JHG, Bertrand D, West SA, Goriacheva II et al (1999). Male-killing Wolbachia in two species of insect. Proc R Soc Lond B 266: 735-740.

Jiggins FM, Hurst GDD, Jiggins CD, Schulenburg JHVD, Majerus MEN (2000). The butterfly Danaus chrysippus is infected by a male-killing Spiroplasma bacterium. Parasitology 120: 439-446.

Juchault P, Frelon M, Bouchon D, Rigaud T (1994). New evi- 
dence for feminizing bacteria in terrestrial isopods: evolutionary implications. CR Acad Sci Paris Sér III 317: 225-230.

Kageyama D, Hoshizaki S, Ishikawa Y (1998). Female-biased sex ratio in the Asian corn borer, Ostrinia furnacalis: evidence for the occurrence of feminizing bacteria in an insect. Heredity 81: 311-316.

Miyahara Y (1984). Abnormal sex ratio in Ostrinia furnacalis Guenée (Lepidoptera: Pyralidae). Jpn J Appl Entomol Zool 28: 131-136 (in Japanese with English summary).

Moreau J, Bertin A, Caubet Y, Rigaud T (2001). Sexual selection in an isopod with Wolbachia-induced sex reversal: males prefer real females. J Evol Biol 14: 388-394.

Ohbayashi F, Suzuki MG, Mita K, Okano K, Shimada T (2001). A homologue of the Drosophila doublesex gene is transcribed into sex-specific mRNA isoforms in the silkworm, Bombyx mori. Comp Biochem Physiol B 128: 145-158.

O'Neill SL, Giordano R, Colbert AME, Karr TL, Robertson HM (1992). 16S rRNA phylogenetic analysis of the bacterial endosymbionts associated with cytoplasmic incompatibility in insects. Proc Natl Acad Sci USA 89: 2699-2702.

O'Neil SL, Hoffmann AA, Werren JH (1997). Influential Passengers: inherited microorganisms and arthropod reproduction, Oxford University Press: Oxford.

Rigaud T, Juchault P (1992). Genetic control of the vertical transmission of a cytoplasmic sex factor in Armadillidium vulgare Latr. (Crustacea, Oniscidae). Heredity 68: 47-52.

Rigaud T, Juchault P, Mocquard JP (1997). The evolution of sex determination in isopod crustaceans. BioEssays 19: 409-416.

Rousset F, Bouchon D, Pintureau B, Juchault P, Solignac M (1992). Wolbachia endosymbionts responsible for various alterations of sexuality in arthropods. Proc $R$ Soc Lond B 250: 91-98.

Schütt C, Nöthiger R (2000). Structure, function and evolution of sex-determining system in Dipteran insects. Development 127: 667-677.

Stouthamer R, Breeuwer JAJ, Luck RF, Werren JH (1993).
Molecular identification of microorganisms associated with parthenogenesis. Nature 361: 66-68.

Stouthamer R, Breeuwer JAJ, Hurst GDD (1999). Wolbachia pipientis: microbial manipulator of arthropod reproduction. Annu Rev Microbiol 53: 71-102.

Swofford DL (1996). PAUP*: phylogenetic analysis using parsimony (and other methods). Ver 4.0. Sunderland, Massachusetts: Sinauer.

Thompson JD, Higgins DG, Gibson TJ (1994). CLUSTAL W: improving the sensitivity of progressive multiple sequence alignment through sequence weighting, positions-specific gap penalties and weight matrix choice. Nucleic Acids Res 22: 4673-4680.

Traut W, Marec F (1996). Sex chromatin in Lepidoptera. Q Rev Biol 71: 239-256.

Vala F, Breeuwer JAJ, Sabelis MW (2000). Wolbachia-induced 'hybrid breakdown' in the two-spotted spider mite Tetranychus urticae Koch. Proc R Soc Lond B 267: 1931-1937.

Vandekerckhove T, Watteyne S, Williems A, Swing JG, Martens J, Gillis M (1999). Phylogenetic analysis of the 16S rDNA of the cytoplasmic bacterium Wolbachia from the novel host Folsomia candida (Hexapoda, Collembola) and its implications for wolbachial taxonomy. FEMS Microbiol Lett 180: 279-286.

Van Meer MMM, Witteveldt J, Stouthamer R (1999). Phylogeny of the arthropod endosymbiont Wolbachia based on the wsp gene. Insect Mol Biol 8: 399-408.

Werren JH, Bartos JD (2001). Recombination in Wolbachia. Curr Biol 11: 431-435.

Werren JH, Nur U, Wu C-I (1988). Selfish genetic elements. Trends Ecol Evol 3: 298-302.

Werren JH, Zhang W, Guo L (1995). Evolution of Wolbachia: reproductive parasites of arthropods. Proc $R$ Soc Lond B 251: 55-63.

Zhou W, Rousset F, O’Neill S (1998). Phylogeny and PCR-based classification of Wolbachia strains using wsp gene sequences. Proc R Soc Lond B 265: 509-515. 